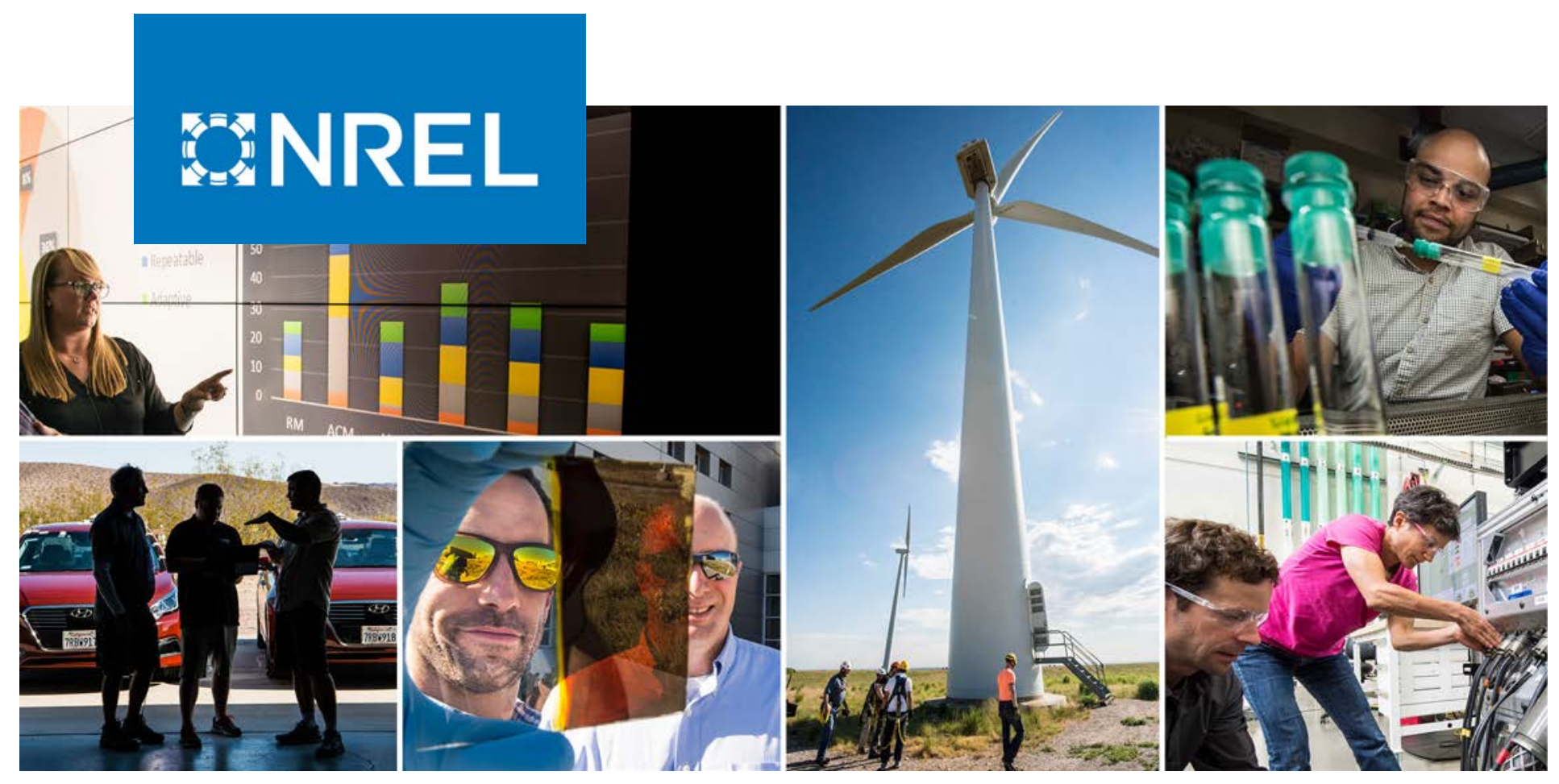

\title{
International Meeting on Fuel Cell and Electrolyzer Quality Control: Summary Report
}

Michael Ulsh, ${ }^{1}$ Michael Hahn, ${ }^{2}$ Francois Girard, ${ }^{3}$ and Ulf Groos ${ }^{4}$

1 National Renewable Energy Laboratory

2 U.S. Department of Energy Hydrogen and Fuel Cell

Technologies Office

3 National Resource Council Canada

4 Fraunhofer Institute for Solar Energy Systems

NREL is a national laboratory of the U.S. Department of Energy

Office of Energy Efficiency \& Renewable Energy

Operated by the Alliance for Sustainable Energy, LLC

This report is available at no cost from the National Renewable Energy Laboratory (NREL) at www.nrel.gov/publications.
Technical Report

NREL/TP-5900-81354

December 2021 


\title{
GNREL
}

\section{International Meeting on Fuel Cell and Electrolyzer Quality Control: Summary Report}

\author{
Michael Ulsh, ${ }^{1}$ Michael Hahn, ${ }^{2}$ Francois Girard, ${ }^{3}$ and \\ Ulf Groos ${ }^{4}$
}

\author{
1 National Renewable Energy Laboratory \\ 2 U.S. Department of Energy Hydrogen and Fuel Cell \\ Technologies Office \\ 3 National Resource Council Canada \\ 4 Fraunhofer Institute for Solar Energy Systems
}

\section{Suggested Citation}

Ulsh, Michael, Michael Hahn, Francois Girard, and Ulf Groos. 2021. International Meeting on Fuel Cell and Electrolyzer Quality Control: Summary Report. Golden, CO: National Renewable Energy Laboratory. NREL/TP-5900-81354.

https://www.nrel.gov/docs/fy22osti/81354.pdf.

NREL is a national laboratory of the U.S. Department of Energy Office of Energy Efficiency \& Renewable Energy Operated by the Alliance for Sustainable Energy, LLC

This report is available at no cost from the National Renewable Energy Laboratory (NREL) at www.nrel.gov/publications.

Contract No. DE-AC36-08GO28308
Technical Report

NREL/TP-5900-81354

December 2021

National Renewable Energy Laboratory 15013 Denver West Parkway Golden, CO 80401

303-275-3000 • www.nrel.gov 


\section{NOTICE}

This work was authored by the National Renewable Energy Laboratory, operated by Alliance for Sustainable Energy, LLC, for the U.S. Department of Energy (DOE) under Contract No. DE-AC36-08GO28308. Funding provided by U.S. Department of Energy Office of Energy Efficiency and Renewable Energy Hydrogen and Fuel Cell Technologies Office. The views expressed herein do not necessarily represent the views of the DOE or the U.S. Government.

This report is available at no cost from the National Renewable Energy Laboratory (NREL) at www.nrel.gov/publications.

U.S. Department of Energy (DOE) reports produced after 1991 and a growing number of pre-1991 documents are available free via www.OSTI.gov.

Cover Photos by Dennis Schroeder: (clockwise, left to right) NREL 51934, NREL 45897, NREL 42160, NREL 45891, NREL 48097, NREL 46526.

NREL prints on paper that contains recycled content. 


\section{Acknowledgments}

The International Meeting on Fuel Cell and Electrolyzer Quality Control was held May 5-6, 2021, as a virtual meeting. The meeting was sponsored by the U.S. Department of Energy (DOE) Hydrogen and Fuel Cell Technologies Office (HFTO).

The organizers would like to gratefully acknowledge Dr. Nancy Garland, formerly of HFTO, for her long-time support and leadership of manufacturing research and development activities and collaborations, including the work at the National Renewable Energy Laboratory (NREL) and this international series of meetings on quality control.

\section{Meeting Organizers}

Michael Ulsh, NREL

François Girard, National Research Council Canada (NRC)

Ulf Groos, Fraunhofer Institute for Solar Energy Systems (ISE)

Michael Hahn, HFTO

Nancy Garland, HFTO

Zachary Taie, HFTO

Peter Rupnowski, NREL

Jesse Adams, HFTO 


\section{List of Acronyms}

DOE

GDL

HFTO

ISE

LTE

MEA

NRC

NREL

OEM

PEM

PGM

PTL

QC

$R \& D$

$\mathrm{R} 2 \mathrm{R}$
U.S. Department of Energy

gas-diffusion layer

Hydrogen and Fuel Cell Technologies Office

Institute for Solar Energy Systems

low-temperature electrolysis

membrane electrode assembly

National Research Council Canada

National Renewable Energy Laboratory

original equipment manufacturer

polymer-electrolyte membrane

platinum group metal

porous transport layer

quality control

research and development

roll-to-roll 


\section{Executive Summary}

Quality control (QC) for both polymer electrolyte membrane fuel cell and electrolysis membrane electrode assembly (MEA) materials is a key challenge for scale-up and cost reduction.

Developing methods for detecting defects, as well as measuring critical material properties and understanding the impact of as-manufactured variations in these materials on cell performance and lifetime, are critical barriers. To help address these needs, the National Research Council Canada (NRC), Fraunhofer Institute for Solar Energy Systems (ISE), and the National Renewable Energy Laboratory (NREL) have organized and facilitated a series of workshops on the topic, bringing together industry, academia, and research institutions from North America and Europe. Prior workshops in Canada and Germany have focused on the status of quality tool capabilities and identification of needed developments for fuel cells. These meetings have garnered an excellent response and follow-on attendance, with over 100 unique attendees.

In fiscal year 2021, NREL, under support from the U.S. Department of Energy (DOE) Hydrogen and Fuel Cell Technologies Office (HFTO), recommended the next meeting in the series of QC workshops be hosted in the United States. Hosting this meeting would provide an increased opportunity for U.S. labs, academia, and especially industry to engage in the ongoing series of meetings, provide insight, and help drive the direction of this international collaborative research effort. It would also maximize the benefit to U.S. industry and U.S. competitiveness for these important technologies. Leveraging the participation and outputs of the previous QC workshops, the major objectives of the proposed U.S.-hosted meeting, in addition to further engagement of U.S. entities, are to:

- Review and discuss the outcomes of the prior QC workshops related to fuel cells and provide opportunity for U.S. industry and stakeholders to comment.

- Add low-temperature electrolysis (LTE) MEA materials to the scope of the workshop series and begin to compare and contrast LTE QC needs with those for fuel cells.

- Prioritize QC needs and output information in a format that can inform HFTO programmatic research, development, and deployment decisions, such as those related to and coordinated with the activities of the new HFTO-supported lab consortia focused on both fuel cells for heavy-duty trucks and low- and high-temperature water electrolysis.

Under HFTO support, NREL, with coordinated facilitation from NRC and Fraunhofer ISE, hosted the international meeting, held on May 5 and 6, 2021. Approximately 80 registrants attended, representing industry, academia, and research institutes. The Day 1 (fuel cells) and Day 2 (LTE) sessions both opened with introductory presentations from labs, DOE, and industry, giving perspectives on overall manufacturing scale-up needs and directions, status of research on the topic, background on the series of international meetings on QC to date, and commentary from industry on QC status and challenges. The Day 2 session was additionally assisted by a presentation on the potential differences between fuel cell and electrolysis materials, structures, and operation that could lead to differences in QC needs and priorities. Presentations were given by the three co-organizing labs and HFTO. From industry, presentations were given on QC status and challenges by $3 \mathrm{M}$ and Gore on fuel cell materials, and by Nel and Plug Power on LTE materials.

On each day, the presentation part of the session was followed by open facilitated discussions to gather feedback from participants on QC status, needs, and challenges for the two technologies. 
On Day 2, an additional open discussion was held to discuss opportunities and mechanisms for collaboration. Excellent participation and engagement from the participants led to a broad range of inputs and suggestions for both technologies, as documented in Section 3 of this report. Overall, the two key outcomes resulting from the discussion were:

- For fuel cells, to engage automotive and system original equipment manufacturers (OEMs) to gather defect and failure data obtained from not only research, but actual system testing and operation, and analyze and publish aggregate results that could lead to better understanding of critical defects and thresholds for quality. This activity potentially could be formatted similar to the long-running HFTO-supported "Technology Validation" projects, where actual system operation and failure data were collected and analyzed, across multiple OEMs, for fuel cell light-duty vehicles, forklifts, and backup power systems. The anonymized aggregation of data across OEMs enabled sensitive data to be used but led to results that were not attributed to the systems or designs of any one OEM, and therefore could be publicly available.

- For LTE, to engage the community, including applicable industry, academia, and labs, to identify, evaluate, and prioritize failure mechanisms for LTE cells. Because manufacturing of and operational experience with LTE MEAs is generally less mature than that of fuel cells (though many similarities in materials and supply chains exist), there is less basis of knowledge to identify key development needs for QC for these materials and processes. An assessment of known failure mechanisms would provide a basis for determining these needs. Several participants were interested in collaborating on this effort.

Going forward, the community and funding agencies will continue to coordinate to initiate these key activities, as well as to seek improved mechanisms for collaboration among disparate organizations and especially across borders. The meeting series is currently planned to continue, with a virtual meeting hosted by Fraunhofer ISE in November 2021. 


\section{Table of Contents}

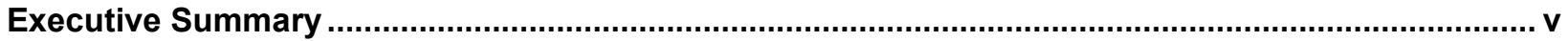

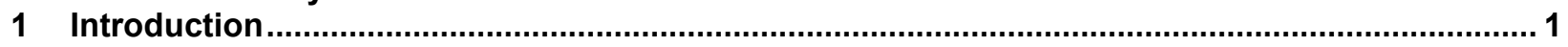

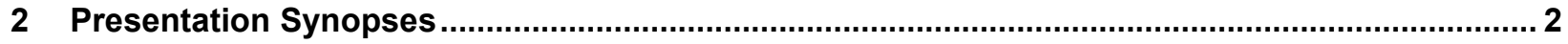

2.1 Welcome, Overview, and Introductory Comments .................................................................

2.2 Industry Commentary on Fuel Cell Quality Control Status and Challenges................................ 3

2.3 Overview of International Research Activities on Quality Control ............................................ 3

2.4 Description and Outcomes of Prior Fuel Cell QC Workshops................................................. 4

2.5 Comparison of Electrolyzer and Fuel Cell Materials, Structures, and Operating Conditions and

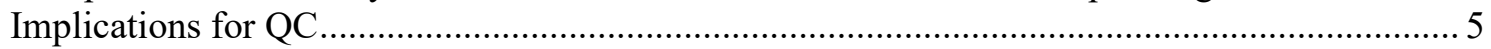

2.6 Industry Commentary on Low-Temperature Electrolysis Quality Control Status and Challenges

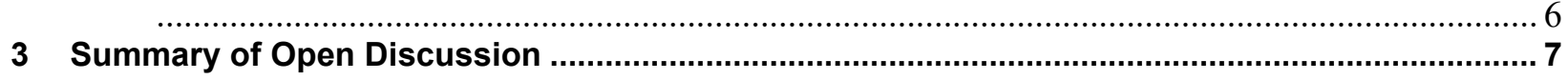

3.1 Low-Temperature Fuel Cell Technologies......................................................................... 7

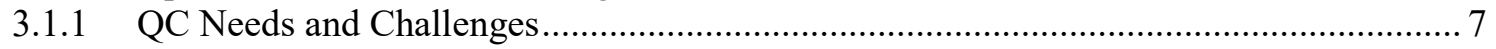

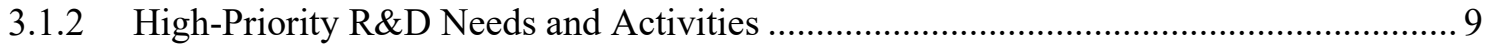

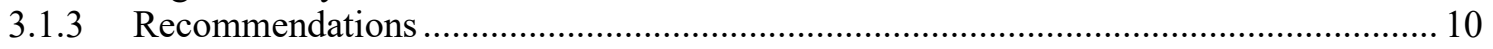

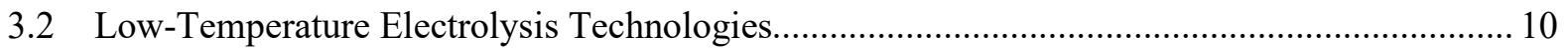

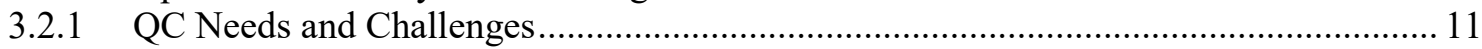

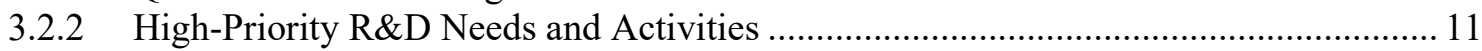

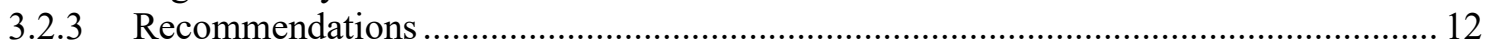

3.3 Collaborative Activities and Mechanisms ........................................................................ 12

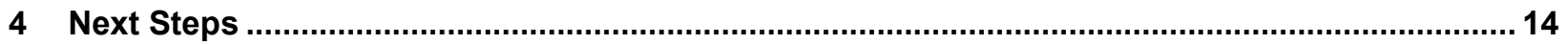




\section{Introduction}

Quality control (QC) for both polymer electrolyte membrane fuel cell and electrolysis membrane electrode assembly (MEA) materials is a key challenge for scale-up and cost reduction of these important U.S. Department of Energy (DOE)-supported energy conversion technologies. Developing both in-line and end-of-line methods for detecting defects, as well as measuring critical material properties and understanding the impact of as-manufactured variations in these materials on cell performance and lifetime, are critical barriers. To address these needs, the National Research Council Canada (NRC), Fraunhofer Institute for Solar Energy Systems (ISE), and National Renewable Energy Laboratory (NREL) have organized and facilitated a series of meetings on the topic, bringing together industry, academia, and research institutions from North America and Europe. Prior workshops in Canada and Germany have focused on the status of quality tool capabilities and identification of needed developments, including:

- The development of detailed function, property, and measurement needs for each MEA component.

- The potential of analytical tools for MEA materials to assist these developments.

- Discussions of possible joint research areas for the interested parties.

There has been a very strong response to the series of collaborative meetings to date, with over 100 total unique attendees participating.

In fiscal year 2021, NREL, under support from the DOE Hydrogen and Fuel Cell Technologies Office (HFTO), recommended the next meeting in the series of QC workshops be hosted in the United States. Hosting this meeting would provide an increased opportunity for U.S. labs, academia, and especially industry to engage in the ongoing series of QC workshops, provide insight, and help drive the direction of this international collaborative research effort. It would also maximize the benefit to U.S. industry and U.S. competitiveness for these important technologies. Leveraging the participation and outputs of the previous QC workshops, the major objectives of the proposed U.S.-hosted meeting, in addition to further engagement of U.S. entities, are to:

- Review and discuss the outcomes of the prior QC workshops related to fuel cells, and provide opportunity for U.S. industry and stakeholders to comment.

- Add low-temperature electrolysis (LTE) MEA materials to the scope of the workshop series and begin to compare and contrast LTE QC needs with those for fuel cells.

- Prioritize QC needs and output information in a format that can inform HFTO programmatic research, development, and deployment decisions, such as those related to and coordinated with the activities of the new HFTO-supported lab consortia focused on both fuel cells for heavy-duty trucks and low- and high-temperature water electrolysis.

Under HFTO support, NREL, with coordinated facilitation from NRC and Fraunhofer ISE, hosted the international meeting, held on May 5 and 6, 2021. The agenda for the two half-day virtual event (provided in Appendix A) included introductory presentations, detailed technical presentations from industry and labs, and open discussion. The open discussion focused on identifying QC technology challenges and needs, ideas and mechanisms for collaborative activities to address needs, and immediate next steps for both low-temperature fuel cell and 
electrolysis MEA materials. Approximately 80 registrants attended, representing industry, academia, and research institutes. The organizations represented by the attendees are listed in Appendix B.

\section{Presentation Synopses}

The Day 1 session on fuel cells opened with welcome and overview presentations, followed by industry commentary on QC status and challenges, presentations on related activities and capabilities, and a review of prior QC workshops by the three co-organizing labs. The Day 2 session opened with a discussion of the similarities and differences between fuel cell and electrolysis MEA materials, structures, and operating conditions, and the potential impact of each on how QC needs and priorities for electrolyzer MEAs might be different than those for fuel cells. This presentation was followed by industry commentary on QC status and challenges, as currently known given the early state of scale-up and commercialization, for electrolyzer MEAs.

This section provides a short summary of each meeting presentation. Presentation materials are available at www.energy.gov/eere/fuelcells/international-meeting-fuel-cell-and-electrolyzerquality-control.

\subsection{Welcome, Overview, and Introductory Comments}

Michael Ulsh of NREL provided welcome remarks and an overview of the workshop. Previous studies have highlighted the importance of QC for automotive fuel cell manufacturing to improve yield and reduce costs. HFTO has funded research and development (R\&D) for in-line QC, and test beds relevant to industry have been developed. Research organizations in Europe and Canada have hosted a series of workshops-with academic, industry, and government participation-to identify and prioritize QC needs for fuel cells. The objective of this workshop is to bring U.S. industry into the discussion and expand the scope to include low-temperatureelectrolyzer materials.

Michael Hahn and Jesse Adams of HFTO provided introductory comments about DOE's manufacturing R\&D activities. Manufacturing is a critical part of DOE's Hydrogen Program Plan, and HFTO has invested in several projects to drive down costs and enable widespread utilization of hydrogen. To take technologies from niche applications to mass markets, industrialscale techniques for manufacturing hydrogen-related technology components are needed. Adding electrolysis to this workshop series fits well with DOE goals to reduce electrolyzer costs and improve durability. DOE's Hydrogen Program Plan identifies the importance of QC to drive electrolyzer cost reductions and help bring about DOE's H2@Scale vision. Processes highlighted in the plan include roll-to-roll processes, technologies for in-line diagnostics, and sensors to detect defects in-line. HFTO has funded about $\$ 20$ million in electrolyzer manufacturing projects in the last half decade and is investing another $\$ 18$ million in the near term in the fuel cell and electrolyzer manufacturing space. 


\subsection{Industry Commentary on Fuel Cell Quality Control Status and Challenges}

Erin Setzler of Gore and Michael Yandrasits of 3M provided industry perspectives on QC status and challenges for fuel cells.

Gore has invested in high-volume, low-cost manufacturing platforms and is committed to ensuring internal product quality by advancing inspection techniques and QC. Gore is working on increasing roll speed and width to improve yield, and currently uses in-line QC methods to detect $x$-y nonuniformities. Future needs may include z-height analysis, detection of pinholes, and possible chemical detection such as batch sampling for iron, as iron is known to enhance degradation. QC needs are determined and prioritized based on practical considerations like cost and speed. The two questions they ask are (1) What is a defect? and (2) How do we detect it? Defects that are currently considered to be potentially harmful include pinholes, thickness variation, silicone, hard materials, Fenton catalyst $(\mathrm{Fe})$, and cations. Gore is also working with university, industry, and national lab partners to conduct studies to identify nonuniformities, track the problems caused in fuel cells over time to determine which defects are important, and reduce the impact of those nonuniformities through improved membrane design and/or manufacturing techniques.

$3 \mathrm{M}$ has developed fuel cell membrane electrode assemblies and catalyst-coated membranes and is now shifting its focus to ionomer technologies. In terms of QC, the goal is to identify defects and eliminate those that result in premature cell failure. Manufacturers don't like the term "defect" because not everything you see is going to result in a negative impact. In the presentation, the term "defect" just identifies a change in attribute. An important consideration for QC is what you're going to do with the information. If you look for something, for example, in optical imaging, you're going to see something. But then what? How do you use this information? Studies using both manual (visual) and automated inspection methods detected high quantities of potential defects. However, in subsequent accelerated stress tests of membranes with known defects, the failure point of the device showed little correlation to the defect location. Other QC considerations not covered in the presentation include electrode and support material defects, camera placement and line speed, data management, rejection strategies, and the impact of operating conditions on failure mode. Conclusions were that inspection is hard, equipment parameters and data analysis can have a large impact on interpretation of the gathered data, debris should be reduced, the impact of defects needs to be understood, and the expectation of zero defects is irrational. The best way to manage all these issues for reliable, high-volume manufacturing is currently not clear.

\subsection{Overview of International Research Activities on Quality Control}

Matthias Klingele of Fraunhofer ISE, Francois Girard of NRC, and Michael Ulsh of NREL summarized respective research activities on quality control at the three co-sponsoring institutes.

Fraunhofer ISE is one of Europe's largest research agencies, and for more than 25 years has conducted fuel cell research and assisted industry in developing fuel cell technologies, specifically low-temperature proton-exchange membrane (PEM) fuel cells for transportation applications. Industry often works with Fraunhofer ISE to optimize certain aspects of a device. Fraunhofer ISE has developed a commercial differential test cell and offers in-house test cells 
that are fully automated and can run 24/7. Fraunhofer ISE's research activities include material optimization (e.g., studying the performance impacts of platinum and ionomer loadings at different current densities), fabrication (e.g., using ex situ analytics to investigate catalyst inks for catalyst-coated membranes), and characterization and degradation analysis (e.g., highthroughput lifetime testing using real drive cycles). In addition to these experimental capabilities for MEAs, Fraunhofer ISE's capabilities include degradation modeling and characterization of other components such as bipolar plate coatings and the gas diffusion layer-bipolar plate interface. Upcoming planned activities at Fraunhofer ISE include work on cell break-in and work to better understand defects, nonuniformities, and tolerances.

$\mathrm{NRC}$ is the main federal research agency in Canada and focuses on problem-solving and economic benefit. NRC initiated its QC R\&D program in 2012 to study fuel cell manufacturing issues. Canada issued a national Hydrogen Strategy in December 2020 focused on creating a domestic market for hydrogen adoption and exporting hydrogen technologies, and this has rekindled hydrogen research activities and investments. From NRC's perspective, QC is important because it reduces parts rejection, ensures product reliability, and provides clear expectations to suppliers, which helps stimulate the supply chain and bring new entrants into the market. NRC focuses on three aspects of supply chain development: manufacturing processes, QC, and system-level analysis. Manufacturing R\&D activities focus on membrane manufacturing, the impact of MEA processes on gas diffusion layer (GDL) properties, and MEA conditioning. QC activities include the international collaboration to understand defect impacts and develop QC tools and methods to detect defects. A new activity involves identification of key defects and methods to introduce them to cell materials and using ex situ and in situ characterization to understand the effects on fuel cell performance and durability. NRC's approach, via its "Book of Attributes" activity, is to determine the properties that need to be measured, determine if there is a measurement technique, and if not, develop one.

NREL has been developing QC technologies for several years with support from HFTO and in partnership with other national labs and industry. NREL's manufacturing research objectives are to (1) detect defects, (2) understand how these defects affect device performance and degradation, and (3) understand how these defects were created during the manufacturing process and mitigate them. NREL is investigating a variety of in-line QC techniques to identify defects of interest in different components and subassemblies. These include membrane thickness imaging using hyperspectral imaging, which works for membranes with and without protective layers or backers; active thermal scanning; simultaneous optical and infrared imaging; spectroscopy-based methods; and optical transmission imaging. NREL also has a unique suite of capabilities for spatially resolved in situ testing to understand the impact of defects on performance and durability, and has performed extensive studies of the effects of membrane and electrode defects.

\subsection{Description and Outcomes of Prior Fuel Cell QC Workshops}

Ulf Groos of Fraunhofer ISE and Francois Girard, Riny Yuan, and Ken Shi of NRC summarized outcomes from prior international workshops on fuel cell QC.

The workshop series began in 2018 at Fraunhofer ISE, but there was strong collaboration with Canada from the beginning. The first workshop focused on in-line detection needs. Follow-on workshops in 2019 identified and prioritized key performance indicators for components and 
measurement technologies and discussed ideas for projects/collaborations. Additional workshops since then have focused on catalyst layers, stacking, and factory acceptance testing. These workshops have included significant industry involvement, with many companies participating throughout the series, and resulted in new collaborations and exchange of information with industry. Project ideas now in development include break-in of fuel cell stacks, end-of-line QC testing, investigation of defect impacts and tolerances, a book of attributes, and an international register of fuel cell components and suppliers.

A key outcome of the first workshop was identifying the need to (1) formalize and harmonize the language for talking about QC (e.g., defining nonuniformity vs. inhomogeneity vs. defect), and (2) elevate the dialogue to common issues, not an individual company's issues or processes, to improve participation and information sharing.

Another outcome was the development and publication ${ }^{1}$ of a "book of attributes" for fuel cell QC, led by NRC. This was a top priority selected out of previous workshops to address a recognized need to prioritize measurements and specifications for QC efforts. The components analyzed include bipolar plates, gaskets and sub-gaskets, catalyst-coated membranes, and gas diffusion layers. For each component, the final book of attributes shows the required functions, associated attributes, properties that control these attributes, method(s) used to test them, groups that are experts in developing those methods, and the priority of technique development. The book of attributes is a living document that will continue to improve and evolve. It sets the stage for what to look at, but continued industry feedback is important to identify critical parameters and tools.

$\mathrm{NRC}$ has also begun an internal research project to identify key defects during PEM fuel cell manufacturing and understand the impact on performance and durability. The team developed a list of all possible defects and their source and then reviewed the list with external consultants (fuel cell developers, component suppliers, and research organizations) to narrow down and prioritize the list. The list of priority defects for membrane, ink, catalyst layer, and GDL was presented. NRC would like to solicit additional feedback from meeting attendees to further prioritize the top 2-3 defects to study for each component.

\subsection{Comparison of Electrolyzer and Fuel Cell Materials, Structures, and Operating Conditions and Implications for QC}

Guido Bender of NREL described NREL's approach to defect assessment and tolerance determination; presented a comparison of fuel cells vs. electrolysis cells, especially in future electrolyzer systems; and summarized some key considerations for adapting fuel cell QC for electrolysis cells.

NREL conducts sensitivity assessments of MEAs having as-manufactured or created defects to determine tolerances and define what a QC system needs to detect. An inhomogeneity becomes a defect when one of three criteria are observed: decreased performance at beginning of life, reduced lifetime, or increased degradation rate. NREL assesses these three criteria through

\footnotetext{
${ }^{1}$ Yuan, Xiao-Zi, et al. 2021. "A review of functions, attributes, properties and measurements for the quality control of proton exchange membrane fuel cell components.” J. Power Sources 491: 229540.

https://doi.org/10.1016/j.jpowsour.2021.229540.
} 
beginning-of-life performance experiments, accelerated stress tests, and drive cycle operation. Effective QC tools need to be able to detect the inhomogeneity below the threshold of where it becomes a defect.

Compared to fuel cell systems, electrolysis systems operate at higher potential $(>1.4 \mathrm{~V})$, and the reaction requires different materials for the anode catalyst and flow fields. In a typical electrolysis cell, the flow field is titanium coated with a platinum group metal (PGM), the diffusion medium, or porous transport layer (PTL), is sintered porous titanium material coated with PGM, and the anode catalyst is unsupported iridium oxide material. The proton conducting membranes in fuel cells and electrolysis cells are similar except for thickness $(<25 \mu \mathrm{m}$ for fuel cells and 90-175 $\mu \mathrm{m}$ for electrolyzers). Current electrolyzer systems are durable but highly overengineered to support niche applications using steady-state operation. Future electrolyzer systems will be operated in dynamic/supply-following applications and have thinner membranes and lower loadings to reduce costs, but will still be expected to have long lifetimes of 80,000 hours or more.

Electrolyzers' high operating potential requires materials with high corrosion resistance, which present new QC needs and challenges. The presentation highlighted several key material and component considerations that are unique to electrolyzers and identified possible QC applications and tools for each. Examples of potential different QC needs included: PTL PGM coating loading and uniformity; loading, uniformity, metal-to-oxide ratio, and in-plane resistance of anode catalyst layer; and recombination layer loading and distribution. In addition, there are stack assembly considerations and challenges that impact LTE lifetimes, including PTL total thickness, sharp edges from cutting PTL materials, variations in PTL and gasket thickness, membrane and gasket creep under high pressure, and membrane swelling resulting in deformation and shearing.

\subsection{Industry Commentary on Low-Temperature Electrolysis Quality Control Status and Challenges}

Adam Paxson of Plug Power and Chris Capuano of Nel Hydrogen provided industry perspectives on QC status and challenges for low-temperature electrolyzers.

Plug Power's stacks have thousands of sealing interfaces and tens of square meters of active area. Due to their high differential pressure, electrolyzer systems are more sensitive to membrane and PTL thickness, and pinholes are more problematic. However, electrolyzers have a lower sensitivity to hot spots because of the liquid water flow. Plug Power is particularly interested in QC to address concerns related to the roughness of the PTL, including protrusions (both anode and cathode) and voids (anode only). Protrusions can push into the membrane and cause harm, and voids can cause high-pressure mechanical stress on the membrane. This potential issue is exacerbated by the desire to go to thinner membranes. There is an unmet QC need for an in-line 3D diagnostic to identify changes in PTL roughness at sufficient resolution to identify defects approaching a critical size.

Nel Hydrogen has developed many large (megawatt-scale) systems operating in the field today. With hundreds of cells and numerous stacks, there are many opportunities for failure, so it is important to minimize defects. Replacing a cell or stack can take a plant down for days or weeks at a time, and given contracted performance guarantees, this amount of downtime could be 
catastrophic. Nel currently manufactures MEAs in-house using batch processes and inspects single parts by hand or via instrument. Nel is exploring roll-to-roll (R2R) MEA fabrication through research projects, and has identified several areas that will need to be managed or monitored through QC inspection processes:

- Coating methods/direct membrane deposition - need to achieve the target size for patch coatings and minimize dimensional changes; need to better control the active area to avoid wasting catalyst or getting catalyst in the seal area.

- Process drift - need a method to identify locations of incomplete coatings and flag the areas with voids that can be recovered by recoating instead of tossing the whole part; need a better understanding of the tolerance bands (percent coated vs. percent incomplete) to define when to reprocess vs. throw away.

- Other standard deformations and active area imperfections (e.g., imprints, wrinkles) — these are currently inspected visually, but could be automated to free up operators' time for other tasks and reduce operator error.

- Sealing area defects - related to deformations in the sealing area or a misalignment of the anode and cathode catalyst layer. For safety, it is important to ensure that catalyst is not within the sealing area to prevent stray electrolysis reactions.

- Realizing the full cost reduction potential associated with R2R manufacturing is only possible with fully automated inspection.

\section{Summary of Open Discussion}

The meeting included three open discussion sessions among all attendees. On Day 1, the discussion focused on QC needs, challenges, and recommendations for fuel cell technologies. On Day 2, the discussion focused first on QC needs, challenges, and recommendations for electrolyzer technologies and then concluded with a discussion of collaborative activities and mechanisms to address the needs and recommendations identified on Days 1 and 2. The following sections summarize the comments from the attendees with regard to QC for fuel cell and electrolyzer MEA materials.

\subsection{Low-Temperature Fuel Cell Technologies}

A significant aspect of the discussion focused on material variations that do and do not impact the functionality of the MEA component and/or full MEA, including the terminology that should be used for the two cases. Various terms were used to indicate an identified local processinduced variation in one of the MEA layers, such as a membrane pinhole or void in an electrode coating, including "feature," "observation," "variation," "inhomogeneity," and "irregularity." This breadth of language was also recognized in the presentations. For consistency within the rest of this report, "feature" will be used to indicate an identified local material variation, regardless of whether or not it has a functional impact. "Defect" will be used specifically to refer to or imply a feature that does unacceptably impact the functionality (i.e., performance or durability) of the MEA.

\subsubsection{QC Needs and Challenges}

The following lists the QC needs and challenges associated with identifying and mitigating defects: 
- What are the features observed during typical MEA component processing, and which features should actually be classified as defects? We need to identify magnitude and frequency of features and map those into an occurrence of failure. What are the thresholds where a feature becomes something that matters?

- What feature size can be tolerated (i.e., is not a defect)? Does this critical size depend on the in situ testing conditions and load profiles? Agglomerates and dust particles should also be considered as features, which might be very difficult to detect in comparison with holes.

- A large percentage of observed features may end up being benign (i.e., not a defect) with respect to durability impacts.

- Knowing which features result in premature failure and then intervening to eliminate them is important.

- A feature for one OEM could be a defect for another, given that system designs and operating conditions will impact the extent to which a feature could become detrimental. Sharing threshold information would be highly useful, but it could require sharing operating conditions and other proprietary information about the manufacturing process.

- How can the fuel cell community talk about needs and challenges related to durability and lifetime without getting into specific processes or materials?

The following lists the QC needs and challenges associated with materials and processes:

- As we move to higher pressure, sealing and gasketing in stacks becomes more important with regard to quality.

- Materials are still new, and we aren't sure yet if we have the tools to measure the properties that are important, or if all relevant properties have been identified.

- Suppliers are ramping up quickly and moving toward fully automated roll-to-roll processes. Materials and components changed completely in the last 10 years, and manufacturing processes, materials, and components will continue to change in the future. It is not clear how these changes will impact the type, magnitude, and frequency of features created. There is a risk of getting locked into specific QC techniques that can't grow/change with the industry.

- To scale up production, high-throughput and high-quality (near-zero fault tolerance) QC methods are needed.

- There is no standard on the QC for MEAs procured from suppliers to ensure that zero MEA defects get into the final assembly.

The following lists the QC needs and challenges associated with in-line vs. end-of-line QC:

- There may be a limitation of what you can see at the end of the line as you build layers. Some things need to be looked at in the moment (i.e., at the point of fabrication on the manufacturing line).

- In-line inspection is preferable, and nondestructive techniques are necessary. The upfront cost can be high, but in-line ultimately works out to be cheaper per unit.

- Any QC check you can do immediately, prior to the next process or operation, is better - that way you can minimize adding value to defected material.

- In general, QC should be implemented after every production step, starting with the control of incoming materials (catalyst powders, membranes) and control of dispersed inks and hence agglomerates in the catalyst inks, as well as defects after coating and drying. 
- In-line QC devices should not only match the high-throughput demand, but also require minimum safety precautions, as safety precautions such as these can be very cost-intensive.

- It's not just about inspection - even if you catch it, can you correct it? If the issue occurs in the middle of the line, how do you correct it without disrupting the process?

- Change in observation rate is a possible metric to use as an alarm to check out a potential problem. This may not identify/correct the problem, but instead intervenes and forces inspection, which can allow for earlier shutdown.

- The amount of data associated with in-line inspection is large. There are significant challenges with (1) simply processing and utilizing a data set this large, and (2) how to use the data to make decisions on what to do with the product.

\subsubsection{High-Priority R\&D Needs and Activities}

The following lists the high-priority R\&D needs and activities to define and categorize a defect vs. a feature:

- Need to better control the in situ studies of features to understand how defects impact durability. What really matters? We need to understand what is harmful or not.

- Need a lifetime durability test; the degradation mechanisms of accelerated stress tests might not be relevant to real-world use. Testing needs to lead to understandings of defect impact on durability, not just performance.

- Expand accelerated and nonaccelerated testing with known or exaggerated features.

- Learn what the process thresholds are that produce defects so manufacturers can eliminate or stay below those thresholds during manufacturing.

- Collect and analyze data to understand the critical limits where features impact devices; incorporate machine learning.

- Categorize and prioritize a set of features that are likely to be fatal defects.

- Publish a study on defect vs. feature identification.

- Conduct experimental validation to determine what are inhomogeneities and identify no-go criteria for the different components.

The following lists the high-priority R\&D needs and activities to reduce the need for QC through process improvements:

- The final goal shouldn't be how to best inspect these components; instead, how can we eliminate the need for inspection?

- Engineer out destructive processing steps so QC isn't as necessary.

- Understand your processes and the way defects come into the product and avoid that.

- Data analytics and machine learning could play a role in assisting to identify and eliminate defect causes from processes.

- Make processes and materials that are less sensitive or more tolerant to defects. At the catalyst layer, a small indentation on the electrode could be a feature or a defect, depending on the substrate. Each OEM could see this differently based on the substrate used. 


\subsubsection{Recommendations}

A key recommendation from the fuel cell discussion was to try to get more defect, failure, and tolerance data from fuel cell OEMs (including automotive OEMs and equipment OEMs) to determine $\mathrm{QC}$ research needs and priorities.

- Prioritize industry interest, as their input carries more weight than that of labs and other $R \& D$ institutions regarding evaluation of research needs and challenges.

- Partner with companies who have been making and operating MEAs for decades and have devices on the road that have been operating for thousands of hours.

- Get agreements with multiple OEMs to gather data and publish aggregate results that can be used to anonymously analyze the sector. This could be modeled after the DOE Technology Validation program's past work with fuel cell electric vehicle and station OEMs and data.

- Contact trade groups that may have broader information on the content of Failure Modes and Effects Analysis and/or can provide connections to OEMs with the most experience.

Other recommendations:

- Leverage knowledge to avoid duplicate efforts at labs and universities - aim for complementary, not duplicate work.

- Identify common issues that research can help solve. For example, it is easier to have open discussions about how — not what—we characterize and measure. Then we can work toward identifying and understanding fundamental property-defect relationships.

- Leverage existing data to create and publish large collaborative data sets. There is a lot of research/inspection data out there, but not a consensus on what to do with it. For example, create a collaborative data set on R2R processing that industry can use to analyze their own processes. Academia can play a role in data analysis.

- Engage experts from other industries with significant QC experience (e.g., batteries, silicon wafers).

- Include equipment manufacturers-for example, if you are using roll-to-roll processes, you should be asking the manufacturers of the R2R machines for feedback about the performance of the machines.

- Apply industry quality programs like Design for Manufacturing and Assembly or Six Sigma to fuel cell manufacturing to help reduce or eliminate inspection needs. Understand how research related to defects and development of QC methods can "talk to" these ubiquitous industry quality methodologies.

- Quantify QC as a standard or benchmark that suppliers can be held responsible to in production.

\subsection{Low-Temperature Electrolysis Technologies}

The discussion on QC for electrolysis materials was heavily informed by the discussion on fuel cells. Generally, the sense was that similar materials will require similar quality assessment. However, as presented on Day 2, it was understood that differences in material properties, cell structures, and operating conditions may require alteration of quality measures, techniques, and methods of study, as compared to fuel cells. In addition, it was understood that the community has less experience at this point with manufacturing and operation of electrolysis materials than 
for fuel cell materials, and thus the levels of understanding of key aspects that will drive quality needs are lower.

\subsubsection{QC Needs and Challenges}

- Similar to fuel cell QC needs, there is a need to understand what is a "feature" vs. a "defect" for electrolyzer materials and components.

- Overdesign is a strategy employed by industry today rather than targeted QC.

- Industry may be resistant to spend resources to change QC methods. Labs can do the work of developing inexpensive but useful QC techniques, but first industry needs to identify the unmet QC needs and failure mechanisms to detect.

- Electrolyzer materials and operating conditions may include new failure modes not applicable for fuel cells:

- Future systems will have thinner membranes and lower loadings that will likely be more susceptible to defects. For example, the uniformity of the PTL protective coating becomes more important as you go to lower loading.

- There is a need to evaluate in situ techniques used to study the impacts of features at pressure. How much learning can we gain without testing at pressure vs. what can you only see in the right way under pressurized operation?

- QC needs for bipolar plates:

- Some similarity to PTL needs for QC.

- Failures are more related to water quality, but that is a material handling/operational failure, not a failure mode for QC to identify.

- Coatings, thickness tolerance (plate itself) for bipolar plates. The base material changes the approach. This is an easier component to tackle for electrolyzers compared to fuel cells as there is more standardization for metals. Some standards are needed for carbon-based plates.

- Regarding in situ testing of features, what is the best way to evaluate performance impacts? Metal iridium materials oxidize and change when operated in a cell. In the lab, we see differences in oxide-to-metal ratios with what is supposed to be the same catalyst in a single batch from a supplier. Not being able to reliably measure Electrochemical Surface Area within a cell is a challenge; indirect measurements are the alternative for now. In situ tools for the study of features in LTE MEA materials are not as mature as for fuel cells - we don't have all the tools we need.

- The big need from industry is scaling up manufacturing to meet forecast demands. This includes automation, R2R processes, and development of new equipment for high-throughput inspection.

\subsubsection{High-Priority R\&D Needs and Activities}

- Electrolysis QC is about where fuel cell QC was several years ago. There is a need to characterize and prioritize failure methods and develop QC testing in the industry.

- Identify the key failures and work with industry on preventative steps.

- Identify the major degradation modes for PEM water electrolyzers (e.g., iridium loss, corrosion of the catalyst layers or porous transport layer, pinholes, delamination, cracking) 
○ Use in situ micro-computed tomography studies to look at degradation. This has been useful for fuel cells and could give great insight into what is happening in electrolysis cells. This also applies to nano-computed tomography.

- Rank MEA components in terms of QC needs (e.g., do the membrane and MEA come first or is PTL coating homogeneity more important?).

- Prioritize failure mechanisms to inform QC requirements and focus QC development efforts. Example inspection needs:

○ PTL roughness - need to ensure absence of detrimental protrusions.

- Interactions between PTL and electrode that lead to degradation.

- Develop a book of attributes for LTE as was done for fuel cells.

\subsubsection{Recommendations}

A key takeaway from the electrolysis QC discussion is that the failure mechanisms for lowtemperature electrolyzer MEA materials must first be identified to inform the determination of QC needs. This is a strong area of emphasis for new activities and consortia, but work needs to be coordinated to align resources and avoid overlap.

Industry participants expressed willingness to provide a high-level analysis of failure modes without giving away details related to intellectual property (e.g., qualitative field failure rates). But it is more difficult to share the criteria by which something is considered a failure. The specifications used to determine failure or poor performance are dictated by operating strategies, and divulging this information could give away competitive advantages.

Participants recommended the formation of a "failure modes working group" to identify the major degradation modes and failure mechanisms, categorize and rank these failure mechanisms in order of importance, and identify research and development needs for novel QC systems for which current methods are insufficient. The scope of the working group should be confined to anything related to the build of a cell or stack that QC can address.

- Organize a follow-up meeting to define and catalog failure modes and create a tiered list of priorities.

- Invite attendees who are or should be involved in understanding failure mechanisms for LTE. Include multiple companies and deployments; it can take a long time for field failures to occur, and the more deployments we have, the better the list becomes.

- Identify and categorize the failure modes of interest.

- Prioritize the list.

- Identify what methods are in use today, and what needs to be developed.

- Look at funding and collaboration mechanisms.

\subsection{Collaborative Activities and Mechanisms}

After the open sessions on fuel cell and electrolysis QC, a discussion was held specifically to identify useful collaborative activities and possible mechanisms to enable those activities. The collaborative community includes industry, academia, and labs - organizations that are partnered, but have different operational objectives as well as different/international funding 
sources that usually can't mix. In this environment, it was understood that collaboration is a goal, but not always facilitated by these externalities.

Collaborative ideas discussed were to:

- Create a database of experts and capabilities for others to call upon. A database for suppliers of inspection equipment would also be helpful.

- Continue to pursue industry input and investment through cost-shared DOE/lab projects.

- Engage with organizations that could assist with developing collaborations or soliciting data:

○ Hydrogen Council.

- Automotive Industry Action Group (AIAG) in North American and Verband der Automobilindustrie (VDA) in Europe.

- Solicit feedback from industry to better understand the criteria for what can be shared in a public fashion vs. what needs to be protected through a non-disclosure agreement or cooperative research and development agreement. What kind of projects would industry support or avoid? Possible ways to solicit this information:

○ Issue a formal DOE Request for Information.

○ Conduct an industry survey.

- Create an industry advisory board.

- Consult other industries such as solar or batteries to see if there are example models of information-sharing.

- Establish collaborations to benchmark devices and level the measurement field, possibly through a new or existing consortium with industry partners. Different labs are working on different defects but using similar platforms. Can those data be shared to get a comprehensive look?

- Improve mechanisms for government agencies to work together and fund activities across borders. There are mechanisms for labs, universities, and industry to work together within borders, but it is harder to have international collaborations, especially from a funding perspective. How can we assist government agencies to work together?

- Continue meetings like this QC series.

- Hold specific coordination meetings among organizations funding this work.

- Form cooperation agreements between countries for coordination of funding.

- Use the National Science Foundation Industry-University Cooperative Research Centers (IUCRC) program as a model for industry to move forward in a collaborative way with universities and government.

- Use OREO as an example of a successful international collaboration between NREL, two universities, and Fraunhofer ISE. Each organization brings its own funding to a common project. Expand the framework of OREO to add more 
entities; an example of effort that could be added to OREO is the in situ research to study the impact of defects.

- Look for organizations that have funding in the same topic area as a starting point for working together - initially working on independent projects but coordinating funding toward the same topic. If there are enough entities working together on the same topic, it may be easier to move that work forward.

- Better understand and develop the supply chain to ensure continuous cost reductions and QC improvements are distributed throughout the supply chain.

\section{Next Steps}

As seen by the breadth of ideas and inputs on recommendations for research activities, as well as methods and needs for collaboration, the community strongly supported further action. Of course, continued prioritization across stakeholders, including industry and funding organizations, is necessary given limited and disparate resources.

Regarding fuel cells, there was a strong sense that, although studies have been done across the community on distinguishing between features and defects, a more coordinated activity is needed, with more emphasis toward impacts on durability. This is especially important for heavy-duty fuel cell applications with much longer lifetime requirements than those for lightduty vehicles. And although labs and academia can provide useful guiding studies, industry, in the form of the automotive and system OEMs, likely already has a huge amount of data on the impact of quality and failures in MEAs that have undergone real system operation. A key outcome of the discussions was the idea to attempt to establish a "Technology Validation"-like activity with OEMs, where potentially sensitive defect and failure data could be shared via anonymization, and aggregate analysis techniques could be used to identify key parameters leading to establishing tolerances and standards, and also pointing toward key additional research that the community could pursue. In a similar vein, reaching out to other more mature industries to learn about data sharing and quality methods was suggested. Coordination across existing studies to reduce overlap and ensure progress toward ultimate goals was another key input, including leveraging current data sets to establish larger knowledge bases.

Regarding electrolyzers, the consensus on the next needed action was clearly a communitywide assessment of degradation/failure mechanisms in operating LTE cells, the results of which would be used to define QC development needs and pathways. Numerous participants indicated willingness to participate in a failure modes working group to pursue this goal, the workplan for which was laid out in Section 3.2.3.

Under its fiscal year 2022 support from HFTO, NREL will coordinate with co-organizers and industry, academic, and other research institutes within the QC workshop community to:

1. Continue to support follow-on workshops, including planning for a next U.S.-hosted workshop, as appropriate.

2. Facilitate the formation of an LTE failure modes working group and a review and initiation of the workplan laid out in Section 3.2.3. 
3. Initiate discussions within HFTO regarding determination of a pathway for pursuit of the key outcome for fuel cells-i.e., interaction with OEMs and integrators with long experience and extensive data on MEA defects and failures toward the exploration of a "Technical Validation"-type aggregated/anonymous data collection and assessment to provide strong validation of further QC development and defect testing needs and directions.

4. Facilitate initial discussions with HFTO and Fraunhofer ISE, potentially then including other relevant organizations (e.g., NRC), to explore the potential to expand the scope and participants of the OREO International Collaboration to include aspects related to the QC workshops.

Further actions will be taken as enabled by available funding and prioritization within HFTO.

In addition to these suggested next steps, there was a clear desire of the community of participants to have mechanisms that better facilitate collaboration, especially across borders. Discussions between funding organizations to further explore potential improved methods was strongly supported. And, as always, DOE and peer organizations in Canada, Germany, and elsewhere can use their convening function to continue to support the gathering of the community at this ongoing series of workshops toward these goals, ultimately contributing toward the acceleration of manufacturing scale-up of these critical clean energy and decarbonization technologies. 


\section{Appendix A. Agenda}

Agenda for the International Meeting on Fuel Cell and Electrolyzer Quality Control, held virtually May 5-6, 2021.

\begin{tabular}{|c|c|c|}
\hline \multicolumn{3}{|c|}{ Wednesday, May 5} \\
\hline Time (EDT) & Topic & Speaker(s) \\
\hline $10: 00-10: 15$ & Welcome and overview of workshop & $\begin{array}{l}\text { Michael Ulsh, NREL } \\
\text { Michael Hahn, HFTO }\end{array}$ \\
\hline $10: 15-10: 30$ & $\begin{array}{l}\text { DOE Hydrogen and Fuel Cells Technology Office } \\
\text { (HFTO) introductory comments }\end{array}$ & $\begin{array}{l}\text { Jesse Adams, } \\
\text { Technology Acceleration } \\
\text { Program Manager, } \\
\text { HFTO }\end{array}$ \\
\hline $10: 30-11: 00$ & $\begin{array}{l}\text { Industry commentary on fuel cell quality control (QC) } \\
\text { status/challenges (15-min. talks) }\end{array}$ & $\begin{array}{l}\text { Erin Setzler, Gore } \\
\text { Michael Yandrasits, } 3 \mathrm{M}\end{array}$ \\
\hline $11: 00-11: 20$ & Overview of Fraunhofer ISE activities & $\begin{array}{l}\text { Matthias Klingele, } \\
\text { Fraunhofer ISE }\end{array}$ \\
\hline $11: 20-11: 40$ & $\begin{array}{l}\text { Overview of National Research Council-Canada (NRC) } \\
\text { activities }\end{array}$ & Francois Girard, NRC \\
\hline $11: 40-12: 00$ & $\begin{array}{l}\text { Overview of National Renewable Energy Laboratory } \\
\text { (NREL) activities }\end{array}$ & Michael Ulsh, NREL \\
\hline $12: 00-12: 15$ & Break & \\
\hline $12: 15-1: 00$ & $\begin{array}{l}\text { Description and outcomes of prior QC workshops (fuel } \\
\text { cells) }\end{array}$ & $\begin{array}{l}\text { Ulf Groos, Fraunhofer } \\
\text { ISE } \\
\text { Francois Girard, NRC } \\
\text { Riny Yuan, NRC } \\
\text { Ken Shi, NRC }\end{array}$ \\
\hline $1: 00-1: 45$ & $\begin{array}{l}\text { Open discussion/feedback on prior findings for fuel } \\
\text { cells }\end{array}$ & Michael Ulsh, NREL \\
\hline $1: 45-2: 00$ & $\begin{array}{l}\text { Open discussion on prioritization of QC R\&D needs for } \\
\text { fuel cells }\end{array}$ & Michael Ulsh, NREL \\
\hline & Adjourn & \\
\hline
\end{tabular}




\begin{tabular}{|c|c|c|}
\hline \multicolumn{3}{|c|}{ Thursday, May 6} \\
\hline Time (EDT) & Topic & Speaker(s) \\
\hline 10:00-10:10 & Welcome and reminder of Day 2 agenda & $\begin{array}{l}\text { Michael Ulsh, NREL } \\
\text { Michael Hahn, HFTO }\end{array}$ \\
\hline $10: 10-10: 30$ & Report-out on Day 1 findings and prioritization & Peter Rupnowski, NREL \\
\hline 10:30-10:50 & $\begin{array}{l}\text { Introductory discussion/comparison of low-temperature } \\
\text { electrolysis (LTE) and fuel cell membrane electrode } \\
\text { assembly (MEA) materials, structures, and operating } \\
\text { conditions and implications for QC }\end{array}$ & Guido Bender, NREL \\
\hline $10: 50-11: 30$ & $\begin{array}{l}\text { Industry commentary on LTE QC status/challenges } \\
(20 \text {-min. talks) }\end{array}$ & $\begin{array}{l}\text { Adam Paxson, Plug } \\
\text { Power } \\
\text { Chris Capuano, Nel }\end{array}$ \\
\hline $11: 30-11: 45$ & Break & \\
\hline $11: 45-12: 45$ & Open discussion on LTE QC needs and challenges & Michael Ulsh, NREL \\
\hline $12: 45-1: 15$ & $\begin{array}{l}\text { Open discussion on prioritization of LTE QC R\&D } \\
\text { needs }\end{array}$ & Michael Ulsh, NREL \\
\hline \multirow[t]{2}{*}{$1: 15-1: 45$} & $\begin{array}{l}\text { Wrap-up discussion: collaboration opportunities and } \\
\text { ideas, final comments }\end{array}$ & Michael Hahn, HFTO \\
\hline & Adjourn & \\
\hline
\end{tabular}




\section{Appendix B. List of Attending Organizations}

The International Meeting on Fuel Cell and Electrolyzer Quality Control included attendees from the following organizations:

- $3 \mathrm{M}$

- Advent Technologies

- Ballard

- Bosch

- Cummins

- De Nora

- Fraunhofer Institute for Solar Energy Systems (ISE)

- General Motors

- Georgia Institute of Technology (Georgia Tech)

- Giner, Inc.

- HyPlat

- Hyzon Motors

- InnoSolve

- Ionomr Innovations Inc.

- Lawrence Berkeley National Laboratory

- Mainstream Engineering

- Mott Corporation

- National Renewable Energy Laboratory

- National Research Council of Canada (NRC)

- Nel Hydrogen

- Nikola Motor

- Plug Power

- Siemens Energy

- The Chemours Company

- The French Alternative Energies and Atomic Energy Commission (CEA)

- Unilia Canada Fuel Cells, Inc.

- University of California Los Angeles

- University of Cape Town

- University of Connecticut

- U.S. Department of Energy

- US Hybrid Corporation

- W.L. Gore \& Associates. 